Archives de sciences sociales des religions

176 | octobre-décembre 2016

Bulletin Bibliographique

Jacqueline Chambron, Lilian Silburn. Une vie mystique

Paris, 2015, Éditions Almora, coll. « Almora Essai », 330 p.

André Padoux

(2) OpenEdition

Journals

Édition électronique

URL : http://journals.openedition.org/assr/28209

DOI : $10.4000 /$ assr.28209

ISSN : $1777-5825$

Éditeur

Éditions de l'EHESS

Édition imprimée

Date de publication : 31 décembre 2016

Pagination : 286

ISSN : 0335-5985

Référence électronique

André Padoux, « Jacqueline Chambron, Lilian Silburn. Une vie mystique », Archives de sciences sociales des religions [En ligne], 176 | octobre-décembre 2016, mis en ligne le 17 juillet 2017, consulté le 24 septembre 2020. URL : http://journals.openedition.org/assr/28209; DOI : https://doi.org/10.4000/ assr.28209

Ce document a été généré automatiquement le 24 septembre 2020.

(c) Archives de sciences sociales des religions 


\section{Jacqueline Chambron, Lilian Silburn. Une vie mystique}

Paris, 2015, Éditions Almora, coll. « Almora Essai », 330 p.

André Padoux

\section{RÉFÉRENCE}

Jacqueline Chambron, Lilian Silburn. Une vie mystique, Paris, 2015, Éditions Almora, coll. « Almora Essai », 330 p.

1 On peut dire que ceux qui s'intéressent à l'Inde - civilisation à bien des égards fascinante - sont de deux sortes. Les spécialistes du sous-continent (indianistes, sanskritistes, anthropologues, etc.) qui en font un objet d'étude scientifique et dont l'approche est objective, détachée. Et ceux, bien plus nombreux, qu'attire l'Inde spirituelle, qui espèrent y trouver une réponse à leur quête, et le maître, le guru, qui leur ouvrira les portes de la Vérité ultime. Ces deux catégories différentes (même si il y a des « zones grises ») ne se mêlent pas. Or le cas de Lilian Silburn, dont le récit de la vie fait l'objet du livre de Jacqueline Chambron est précisément celui d'une personne qui a su, semble-t-il, mener de front, vivre, « exister » les deux approches de l'Inde que nous avons distinguées, avec autant de succès et de facilité, sans les mêler, mais en les faisant s'enrichir mutuellement. Elle a en outre donné à l'une d'elles, la "mystique ", une présence sociale. En effet, chercheur indianiste ayant fait toute sa carrière au CNRS, elle a aussi cherché puis trouvé en Inde un maître spirituel qui lui a fait connaître une expérience mystique d'une intensité et profondeur exceptionnelles qui a complètement transformé sa vie sans qu'elle cesse pour autant d'être une indianiste de qualité généralement reconnue comme telle par ses pairs. Son cas mérite donc d'être présenté ici, car, outre son intérêt propre, il pose un double problème. Celui de la coexistence chez un même être de deux natures, pourrait-on dire, celle de chercheur et celle de mystique. Et celui que peut poser le fait que cette mystique exceptionnelle (dont plusieurs des collaborateurs des Archives ont connu l'existence ou l'ont 
approchée) resta ignorée de la plupart de ceux qui l'ont connue et même de ceux qui lui ont été longuement proches. Car ce double aspect de sa personnalité se retrouvait dans la vie courante, où elle était pour ceux qui la connaissaient une personne très gaie, ouverte, soucieuse des autres, prête à les aider, dont on savait qu'elle avait un accent spirituel important, mais sans avoir idée de la nature exceptionnelle de ce qu'elle avait connu et « vivait ». Pour eux, le livre de Chambron fut à cet égard une vraie découverte, un choc, même.

2 Ce livre - pour en venir à lui - est un document. Il décrit la vie de Lilian Silburn de son enfance à ses derniers jours (en 1993), mais aussi et principalement la relation spirituelle d'une nature existentielle profonde qui la lia toute sa vie à son guru, luimême d'une sorte peu courante. C'est à la description de ce lien et à ce qu'il impliquait existentiellement qu'est pour l'essentiel consacré cet ouvrage qui, tout en décrivant sa vie, donne des extraits du journal qu'elle tenait à la demande de son guru, comme de leur correspondance, des notes personnelles et autres documents ainsi que des témoignages de diverses sortes.

Lilian Silburn est née à Paris en 1908 d'une mère française et d'un père britannique : elle avait les deux nationalités. Elle eut dès son enfance le sentiment de la grâce divine et des sortes d'extases, mais ce fut une enfant puis une adolescente active, enjouée, déjà bonne nageuse, bien intégrée dans le monde. Elle était aussi très indépendante, ayant le goût des grands espaces et de la solitude. Elle aurait voulu entrer en religion, mais ses parents s'y opposant, elle opta pour des études de philosophie (suivies de 1938 à 1948), pensant qu'elles l'aideraient à approcher la Vérité ultime à laquelle elle aspirait. Étudiante brillante, elle fut, à la Sorbonne, assez proche de Gaston Bachelard, mais elle s'orienta tôt vers la pensée de l'Inde, apprenant le sanskrit, le pâli (et même l'avestique) suivant l'enseignement de Louis Renou, de Paul Fouché et de Paul Masson-Oursel. Elle s'intéressa alors particulièrement au śivaïsme du Cachemire - et dès lors au tantrisme choix original à cette époque où ces deux sujets étaient pratiquement ignorés de l'Université. Elle acheva ses études avec un diplôme de l'École Pratique des Hautes Études qui était une traduction annotée de la Śivarūtravimarśinī de Kṣemarāja, texte śivaite tantrique. En 1947, paraissait son étude sur le śivaisme du Cachemire et le tantrisme dans le premier volume de L'Inde classique. Elle rédigeait alors aussi, dans un domaine tout différent, sa thèse de doctorat, Instant et Cause, Le discontinu dans la pensée philosophique de l'Inde (parue seulement en 1955). Elle entra alors au CNRS où elle fit toute sa carrière d'indianiste. Qu'elle se soit attachée à l'étude du śivaisme cachemirien apparaît comme grandement justifié, car l'ensemble de ces textes (élaborés entre le VIII et le XIV siècles de notre ère), représente certainement ce que l'Inde a produit de plus remarquable, de plus brillamment intelligent: on le sait maintenant depuis que, notamment sous l'impulsion d'Alexis Sanderson, professeur à Oxford, cette tradition dans ses diverses formes a été systématiquement étudiée. Ses travaux furent pionniers à cet égard.

4 Il faut ajouter ici que Lilian Silburn, qui semble avoir eu un talent inné de graphologue, avait élaboré avec son amie Anne-Marie Esnoul, collaboratrice de Louis Renou et enseignante de sanskrit à la Sorbonne, une étude des «types » fondée sur l'observation de la morphologie en liaison avec le caractère. Elle accumulait les observations dans ce domaine. On a pu la voir ainsi saisir intuitivement le caractère, la nature, d'une personne par la vue de ses traits, de son écriture, d'un de ses gestes, même. Noter cela ici n'est pas sans intérêt, car c'est un trait profond de sa nature et qui explique certains 
aspects ou moments de son comportement, dans ce cas, à la fois observateur et mystique.

Désirant approfondir sa connaissance de cet ensemble de traditions qui allait être le sujet/l'objet de sa recherche pendant toute sa vie, Lilian Silburn part en mission d'étude en Inde en 1949 pour y travailler avec le swami Lakshman Joo (1907-1993), le dernier représentant de la tradition śivaite du Trika qu'exposa notamment ce maître exceptionnel que fut Abhinavagupta (fin $\mathrm{x}^{\mathrm{e}}$ - début $\mathrm{xI}^{\mathrm{e}} \mathrm{s}$.). Elle se rendit auprès de ce swami, habitant dans des conditions difficiles non loin de son ashram. Elle devait par la suite y passer à divers moments de longs mois jusqu'en 1961 en étudiant avec lui notamment le Tantrāloka, d'Abhinavagupta, grand traité sanskrit qui est un texte de base pour la connaissance du śivaisme tantrique. Elle le traduisit entièrement. Mais elle espérait aussi, plus fondamentalement, trouver en Inde un maître spirituel qui lui ouvrirait une voie vers Dieu, au-delà de toute forme religieuse constituée. Elle la trouva dans la ville de Kanpur où elle alla sans aucune intention préméditée et même "à l'encontre de (ma) volonté » auprès d'un maître soufi Radha Mohan Lal Adhauliya - un Kayasth, un hindou, donc, pas un musulman (mais les Kayasth étaient traditionnellement au service de Moghols), initié dans une branche régionale de la lignée soufie Naksbandhiya par son père et par un "Grand Soufi ", dont elle reconnut immédiatement la nature exceptionnelle et par qui elle allait vivre une expérience mystique transformante faisant d'elle désormais un être nouveau. Détail caractéristique, elle dit que ce qui la frappa alors, ce fut « l'extraordinaire écriture du Guru et de son frère et celle, merveilleuse du Soufi ». Auprès de ces maîtres, elle tomba, dit-elle, dans " une simplicité de silence dont [elle] n'avait jamais rêvé ", «toutes les structures sont tombées ». Mais, toute transformée qu'elle se sentît alors, elle n'en perdit pas son bon sens de chercheur et, en dépit de la confiance totale qu'elle avait dans le Guru, elle le mit pendant plusieurs jours à l'épreuve en le taquinant, plaisantant, épreuve dont il triompha grâce à son " profond sens de l'humour ». Mais, en même temps, elle vivait intérieurement ce que le guru lui avait donné. Le quittant après quelques jours, elle partit pour le Kumbh Mela, grand pèlerinage hindou qui se tenait alors à Hardwar, au bord du Gange ; elle y prit part. Mais, le troisième jour, perdue dans la forêt, elle ne voyait plus rien du Mela, car "une nouvelle vie commençait... ce jour, le vrai jour de [sa] naissance ». Ce matin-là, elle nagea au milieu du Gange dans le courant puissant irrésistible d'un torrent de montagne débouchant sur la plaine. Puis elle se laissa emporter par le courant étant dans un état mystique profond. Avec peine, elle sortit du fleuve à demi consciente. Pendant quinze jours et quinze nuits elle parcourut la forêt, dormant sous les arbres, mangeant ce qu'un sannyasi (un ascète) nu lui donnait, oubliant tout: "j'étais complètement ivre et à moitié perdue». On ne peut pas rapporter ici le récit qu'elle fit de ces jours (et d'ailleurs de sa vie jusqu'alors) dans une conférence (p. 43-54) qu'elle fit peu après à la demande du guru devant un groupe de ses disciples. Elle y décrit - dans la mesure où cela peut se faire - les caractéristiques de l'état mystique où elle se trouve alors : une douceur délicieuse à la fois spirituelle et corporelle «mais qui n'est pas un état du monde », des coups au cœur " comme si les cavernes du cœur (hrdguha) étaient creuses et qu'une immense puissance les remplissait», "une félicité si excessive que je ne pouvais supporter plus d'une seconde ». À cette époque elle vivait parfois des états de "sommeil yogique " (yoganidra) où on ne dort pas, mais où on est sans conscience du monde. De tels états (et d'autres) sont décrits dans des extraits de son journal et dans sa correspondance avec le guru et avec quelques proches. Elle revint vers le guru qui lui 
fit vivre avec force et profondeur des états mystiques intenses, jours d'autant plus épuisants que ses conditions de vie à Kanpur étaient sommaires et très inconfortables. Elle rentra épuisée en France en juin 1951. Elle y rentra toutefois sans perdre contact avec le Guru, car elle conservait un lien spirituel constant avec lui: ils « communiquaient » même quand elle était en France, cela jusqu'à la mort du Guru en 1966. Ce lien existentiel permanent avec son guru - qu'elle " vivait », « existait »-, trait caractéristique de sa vie, est le thème même de ce livre et peut en faire l'intérêt dans le cadre de nos Archives.

6 Le guru, quant à lui, considérait Lilian comme la plus importante de ses disciples (la plus proche, peut-être) dont le rôle était désormais, à ses yeux, de faire connaître en France son enseignement. C'est un point sur lequel il revient souvent dans sa correspondance avec elle, de 1951 à 1966 dont des extraits sont donnés dans la section «Lilian et le guru 1950-1966» (p. 69-198), qui en est la portion la plus intéressante. Elle l'est moins par ce qui nous est donné de la correspondance du guru (l'essentiel entre eux ne passait pas par lettres) qui est spirituellement assez conventionnel et mentionne beaucoup ses difficultés matérielles et ses graves problèmes de santé. Mais avant tout par la contribution de Lilian, faite d'extraits de sa correspondance avec le guru, avec des (spirituellement) proches ou des amis, mais surtout de son journal et de notes personnelles: cela forme un ensemble remarquablement intéressant sur les expériences comme sur la vie du mystique, qui a des moments de lumière incomparable, mais aussi de nuit et de désespoir. On a là, à mon sens - je ne suis pas mystique -, sur ce domaine un document d'une qualité rare, car rares sont, je crois, les mystiques capables de se voir vivre en tant que tels - et de le décrire lucidement. Signe parmi d'autres de cette attitude : sollicitée, en 1971 par deux chercheurs neurologues, Henrotte, du CNRS et Etéveneu, de l'INSERM, de laisser enregistrer ses ondes cérébrales dans un état mystique profond, elle s'y prêta, curieuse et amusée. Les résultats de l'expérience furent publiés dans La Recherche («Les états de conscience modifiés volontairement ») en décembre 1972.

7 Ces traits se retrouvent, différemment, dans la section suivante du livre, «La vie au Vésinet » (p. 215-285). Revenue en France en 1951 après son expérience transformante d'octobre 1950, Lilian Silburn s'attacha à réaliser la tâche que lui avait confiée son guru : faire connaître en France son enseignement. Elle donna l'initiation à quelques (rares) personnes qui en étaient « capables » - dont Chambron - et, sur un plan moins élevé, en réunissait d'autres, un petit groupe, chez elle, au Vésinet, une ou deux aprèsmidi par semaine, avec en outre des rassemblements annuels (les bandhara) consacrés au guru dont la présence spirituelle est censée être alors spécialement présente, rassemblant un grand nombre de participants celui-ci croissant jusqu'à plusieurs douzaines (certains venant de province, de Marseille notamment où un petit groupe s'était formé autour d'un disciple de Lilian). Aux réunions de l'après-midi, des personnalités étrangères (Durkheim, Mgr Scrima...) participaient parfois. Cela jusqu'à son décès en 1993 (la pratique étant alors reprise jusqu'à nos jours, chez elle au Vésinet, par Chambron). Ainsi s'est créé, prenant une dimension sociale, en région parisienne, mais (avec quelques participants de province) non pas une secte, un mouvement spirituel assurant la présence en France de l'enseignement du guru de Kanpur avec ce que Lilian Siburn a pu lui apporter spirituellement. Cette transmission s'y fait « de cœur à cœur » par la présence spirituelle, silencieuse du maître, sans aucun rite, selon la tradition soufie. Voie directe d'accès à Dieu, celle-ci n'implique aucune référence, textuelle ou autre, à l'Inde, que certains participants actuels ne doivent d'ailleurs pas 
connaître. Tout cela est décrit, avec divers documents, dans cette section qui est illustrée de nombre de photographies, certaines en couleur montrant Lilian et ses proches au Vésinet ou ailleurs.

8 À partir de son retour en France, Lilian Silburn poursuit parallèlement son travail de chercheur au CNRS en rédigeant et publiant la traduction abondamment présentée et commentée de neuf textes sanskrits du śivaisme du Cachemire, allant du Paramārthasāra, de Abhinavagupta (1959) aux Spandakārikā (1990), avec un volume (Hymnes aux Kāli. La roue des Énergies divines) sur l'école « séquentielle» peu connue du Krama, tous parus dans la collection des «Publications de l'Institut de Civilisation Indienne de l'Université de Paris ». À la fin de sa vie, elle voulut faire paraitre la traduction qu'elle avait faite avec le swami Lakshman Joo des chapitres 2 à 5 du Tantrāloka, sur les voies de la libération; j'avais pour ma part traduit le chapitre et devais par la suite rédiger l'introduction et mettre au point l'ouvrage paru en 1995. Elle $\mathrm{y}$ travailla jusqu'à ses dernières semaines. Elle était alors toujours intellectuellement active, en même temps que d'une puissance spirituelle intense, mais on sentait, parfois vivement qu'elle était aussi, essentiellement, « ailleurs ». C'est en ce même état qu'elle fut présente trois semaines avant son décès à la grande réunion annuelle, le bhandara, de cette année-là recevant individuellement tous les participants - une action courageuse montrant sa fidélité à la mission que lui avait confiée son guru.

Pour ses traductions commentées, où le " commentaire » occupait généralement plus de place que le texte traduit, Lilian Silburn agissait évidemment en indianiste, sa maitrise du sanskrit - dont le vocabulaire métaphysique et mystique est beaucoup plus riche que le nôtre - lui permettant de déchiffrer des textes souvent difficiles. Elle y utilisait toujours la terminologie même de ces textes en sanskrit, terminologie qui en était venue à être le mode d'expression de sa pensée scientifique - cela notamment pour décrire des états mystiques inévitablement présents dans des textes visant à acheminer les adeptes vers le salut. Mais pour ces états-là, c'est ce qu'elle devait à ce que son guru lui avait fait connaître qui nourrissait sa pensée et sa pénétration de la visée profonde du texte. Cela apparaît dans tout ce qu'elle a écrit, mais peut-être plus particulièrement dans sa présentation du Vijjñānabhairava parue en 1961. Cet extraordinaire texte du $\mathrm{vIII}^{\mathrm{e}}$ siècle, typiquement tantrique par le recours à tout ce qui de l'être humain relève du corps et de ses pulsions, expose 112 moyens, pour un yogin expert, d'atteindre directement l'Absolu notamment en laissant la place à une pulsion puis en la transcendant. Lilian Silburn les explique tous, puis, dans une longue "Postface ", "Les cycles de la progression mystique », elle s'attache à montrer que ce texte laisse apparaître onze cycles. Cet ouvrage est la plus connue de ses œuvres. Plusieurs fois réédité, il a un certain impact: on le trouve utilisé par des hypnothérapeutes. Il a amené plusieurs personnes à Silburn. Il est actuellement traduit en Argentine où il est parfois utilisé dans des réunions spirituelles, les organisateurs étant en rapport avec Chambron. Un groupe d'une dizaine d'adeptes s'est également formé au Canada, à Ottawa : autres cas de la présence sociale de Silburn.

Le livre s'achève par un chapitre, "La dimension mystique ", composé de textes rédigés par Lilian Silburn sur divers thèmes, notamment sur le rôle du guru : une sorte de petit traité de la vie mystique. On a dans cet ouvrage, à l'occasion de la découverte d'une personnalité à bien des égards exceptionnelle, un ensemble documentaire intéressant tant sur le phénomène mystique que sur la façon dont celui-ci peut être vécu et être socialement présent. 
11 Sur la tradition dont relève Radha Mohan Lal Adhauliya, voir R.K. Gupta, Yogis in Silence. The Great Sufi Masters (Delhi, B.R. Publishing Corporation), ouvrage très typique de la dévotion indienne aux gurus. Bien différemment, dans Thomas Dahnahrdt, Change and Continuity in Indian Sufism (Delhi, D.K. Printworld, 2002), un chapitre examine de façon critique la tradition soufie régionale dont relève ce guru. 